\title{
Comparaison n'est pas raison
}

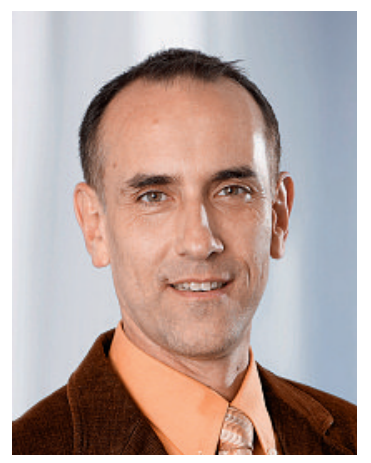

ter qu'ils n'amènent ni clarté ni transparence.

Les évaluations établies d'après le taux de mortalité ne convainquent pas pour la simple et bonne raison que les chiffres ne sont pas adaptés aux risques et qu'ils subissent des variations aléatoires importantes. Tant que les profils de risque des patients ne seront pas connus de manière précise, il sera essentiel de n'interpréter les registres et comparaisons de mortalité qu'avec une extrême prudence.

Ce n'est qu'au moment où l'on disposera de données telles que l'âge, l'état général, la co-morbidité, etc. que l'on pourra prévoir la mortalité en fonction du risque. Cette prévision pourra ensuite être comparée au taux de mortalité effectif. Dans le cas contraire, les chiffres retiennent faussement l'attention et pour le dire sobrement - conduiront à un sentiment de malaise auprès de la population en général et des patients en particulier.

Cette question épineuse a été examinée dans le cadre d'une étude révélatrice publiée par deux épidémiologistes dans le British Medical Journal [1]. Richard Lilford et Peter Pronovost sont parvenus à la conclusion que les taux de

\section{La qualité des hôpitaux ne peut pas être déterminée d'après le taux de mortalité.}

mortalité n'étaient que très rarement pertinents dans les comparaisons croisées entre hôpitaux. Ces chiffres n'expliquent guère les causes de mortalité, alors qu'ils stigmatisent et provoquent des sentiments d'impuissance et de frustration auprès de toutes les parties impliquées. Selon les auteurs, la popularité de tels chiffres s'explique par le besoin des décideurs politiques de disposer de standards de qualité au premier abord clairs et faciles à mesurer. Les deux spécialistes plaident en faveur de systèmes d'évaluation rigoureux et reproductibles pour mesurer les processus hospitaliers internes, afin de réduire les erreurs à l'avenir et de pouvoir effectuer sur cette base des comparaisons équitables entre les hôpitaux. Cela me semble être une bonne démarche qu'on ne peut que soutenir.

Les systèmes d'assurance-qualité qui garantissent que les prestations hospitalières sont bonnes (diagnostic, traitement, soins et prise en charge), et qu'elles le resteront, en disent beaucoup plus long sur l'évaluation d'un hôpital. Citons notamment les processus standardisés sous forme de SOP («standard operating procedures») écrits, qui sont systématiquement contrôlés et garantis, par exemple au moyen

\section{Les processus hospitaliers internes jouent un rôle déterminant pour la qualité.}

de certification et d'accréditation. Ce qui est valable au niveau d'un hôpital l'est aussi pour la pertinence des comparaisons entre médecins. L'évaluation et la comparaison des prestations des médecins hospitaliers sont tout aussi dénuées de sens lorsqu'elles excluent les conditions-cadres centrales. Dans la plupart des cas, il faudrait évaluer les prestations de l'équipe et prendre en compte l'ensemble des processus.

En matière de qualité, l'enseignement médical représente un appui supplémentaire dont l'importance en milieu hospitalier ne doit pas être sous-estimée. En effet, l'enseignement n'est pas uniquement «un facteur de coûts»: celui qui forme doit constamment évaluer son activité de manière critique et rester au meilleur niveau de connaissances.

Le fait qu'en Suisse, il y ait proportionnellement bien plus de personnes qui meurent à l'hôpital qu'ailleurs, est connu. Cela ne dépend toutefois pas des soins médicaux mais de l'état de santé des patients, souvent trop gravement malades ou grièvement blessés pour survivre. Pour les médecins, il est magnifique de sauver la vie d'un patient gravement atteint, mais mourir fait également partie du cycle de la vie.

Pour un pessimiste, le grand danger des futures évaluations d'hôpitaux consisterait à ce que les opérations à risque, susceptibles d'influencer négativement la statistique de mortalité, ne soient plus réalisées sur place, et que les risques soient ainsi transférés ailleurs. Mais si mourir dans les hôpitaux suisses devenait un indicateur de qualité et si, par là même, les cas de décès ordinaires et quotidiens devenaient brusquement un problème d'image, nous aurions alors un véritable problème!

Dr Gert Printzen, membre du Comité central, responsable du domaine Produits thérapeutiques et du domaine Médecins salariés

1 Lilford R, Pronovost P. Using hospital mortality rates to judge hospital performance: a bad idea that just won't go away. BMJ. 2010;340:955-57. 